OPEN ACCESS

Edited by:

Andrea Mari,

Careggi University Hospital, Italy

Reviewed by:

Eric Chung,

Consultant, Brisbane, QL, Australia

Enrico Checcucci,

Fondazione del Piemonte per

l'Oncologia, Istituto di Candiolo

(IRCCS), Italy

*Correspondence:

Lu Chen

cl12063@rih.com.cn

Danfeng Xu

xdf12036@rih.com.cn

${ }^{t}$ These authors have contributed equally to this work

Specialty section: This article was submitted to Genitourinary Oncology, a section of the journal

Frontiers in Oncology

Received: 17 May 2021

Accepted: 19 July 2021

Published: 05 August 2021

Citation:

Liu A, Gao Y, Huang H, Yang X, Lin W, Chen $L$ and $X u D$ (2021) A Combined Technology to Protect the Anatomic Integrity of Distal Urethral Sphincter Complex in Radical Prostatectomy Improves Early Urinary Continence

Recovery Without Sacrifice of Oncological Outcomes.

Front. Oncol. 11:711093. doi: 10.3389/fonc.2021.711093

\section{A Combined Technology to Protect the Anatomic Integrity of Distal Urethral Sphincter Complex in Radical Prostatectomy Improves Early Urinary Continence Recovery Without Sacrifice of Oncological Outcomes}

\author{
Ao $\mathrm{Liu}^{1 \dagger}$, Yi Gao ${ }^{1 \dagger}$, Hai Huang ${ }^{1 \dagger}$, Xiaoqun Yang ${ }^{2}$, Wenhao Lin $^{1}$, Lu Chen ${ }^{1 *}$ \\ and Danfeng $\mathrm{Xu}{ }^{1 *}$ \\ ${ }^{1}$ Department of Urinary, Ruijin Hospital, Shanghai Jiaotong University School of Medicine, Shanghai, China, ${ }^{2}$ Department of \\ Pathology, Ruijin Hospital, Shanghai Jiaotong University School of Medicine, Shanghai, China
}

Purpose: Our primary aim was to present a combined technique to protect the anatomic integrity of distal urethral sphincter complex (DUSC) during minimally invasive radical prostatectomy $(\mathrm{RP})$ and discuss its impact on urinary continence (UC) recovery. The second aim was to define the learning curve of the combined technique.

Methods: We conducted a non-randomized retrospective study. There were 314 consecutive patients who received RP by the same urologist surgeon with more than 2,000 prior cases in Shanghai Ruijin Hospital between March 2017 and April 2020. Included in this study were 263 patients with clinical T1-T2 stage. We modified a combined RP (Comb-RP) technique including endopelvic fascia no-incising technique, dorsal venous complex (DVC) no-ligation technique, intrafascial dissection technique, and anterior reconstruction technique so as to preserve the anatomic integrity of DUSC. The patients were assigned to two groups: a Comb-RP group and a conventional RP (Conv$\mathrm{RP}$ ) group. Continence rates were assessed every 3 months after removal of the catheter. UC was defined as 0 pad per day. Peri-operative variables of the patient including operation time, estimated blood lost (EBL), positive surgical margin (PSM), and postoperative complications were also collected. Scatter-graphs of learning curves were drawn using locally weighted scatterplot smoothing (LOWESS).

Results: RP was accomplished smoothly in all 263 cases. The pad-free UC rates in ConvRP group and Comb-RP group were 17.3 vs. $27.8 \%(P=0.048)$ at the removal of the catheter, 35.8 vs. $50.0 \%(P=0.027)$ at 1 month, 60.5 vs. $76.1 \%(P=0.012)$ at 3 months, 87.7 vs. $96.5 \%(P=0.022)$ at 6 months, and 94.7 vs. $97.7 \%(P=0.343)$ at 12 months. Kaplan-Meier analysis showed significantly higher and faster continence recovery in the 
Comb-RP group (mean 4.9 vs. 2.6 months, Log Rank $P=0.001$ ). There was no significant difference in PSM rate between the Comb-RP and Conv-RP group (31.1 vs. $31.2 \%, P=0.986)$. The learning curves of peri-operative variables, oncological and functional outcomes achieved the lowest point or plateau at the 20th-60th cases.

Conclusions: The anatomic integrity and intact pelvic floor interplay of DUSC is important for its function. Our combined technique was a safe and feasible technique for improving early UC in RP with no significantly increased PSM rate and no significant difference in long-term UC.

Keywords: radical prostatectomy, urinary incontinence, neurovascular bundle, urinary sphincter, reconstruction, learning curve

\section{INTRODUCTION}

Radical prostatectomy (RP) is one of the most important methods for the treatment of localized prostate cancer $(\mathrm{PCa})$. On the premise of ensuring the effect of tumor control, protection of urinary continence (UC) has long been the crux in RP. Various techniques for early UC recovery have emerged in recent years. The early UC recovery rate of the above techniques is reportedly 23-84\% (1-8). Among different UC theories, the urethral sphincter has been widely accepted as a significant factor for improving early continence. In addition to the functional-length, the anatomic fixation and integrity of the urethral sphincter are essential to preserving UC after RP (4).

The UC structure is a complex systematic integrity rather a simple combination of individual anatomic structures, which was named as distal urethral sphincter complex (DUSC). In Campbell-Walsh Urology, the DUSC refers to the UC structure from the colliculus seminalis extending to the proximal bulbar urethra, including the prostatic urethra, membranous urethra (MU), smooth muscle sphincter of prostatic and membranous urethra, prostatic striated sphincter (PSS), membranous urethra striated sphincter (MUS), periurethral striated sphincter (PUSS), puboprostatic ligaments, and other pelvic connective tissues. The key point of this concept lies in the definition of the complex. It is not a simple physical superposition of multiple anatomic elements; rather, it is an organic integrity in which these anatomic elements link with each other and affect each other. In surgical practice, preservation of the DUSC means maintaining the integrity of these structures and protecting them from being damaged.

As we continually try to improve our technique, we adopt endopelvic fascia no-incising technique, dorsal venous complex (DVC) no-ligation technique, intrafascial dissection technique, and anterior reconstruction technique so as to preserve the anatomic integrity of DUSC as much as possible to ensure its function. We postulate these approaches are key steps to maintain the anatomic integrity and intact pelvic floor interplay of DUSC and can be made smoothly in both robotic and laparoscopy assisted RP.

In the current paper, we described our combined technique and attempted to compare differences between the combined RP (Comb-RP) and the conventional RP (Conv-RP) in terms of the peri-operative data and UC recovery. We also evaluated the effect of surgical experience on peri-operative, functional, and oncological outcomes in the Comb-RP group.

\section{PATIENTS AND METHODS}

\section{Study Design and Patient Selection}

This was a non-randomized retrospective study. There were 314 patients who received RP by the same urologist surgeon with more than 2,000 prior cases in Ruijin Hospital (Shanghai, China) between March 2017 and April 2020. The inclusion criteria were patients who met the RP indications with clinical T1-2 stages. A pre-surgery prostatic MRI was performed to identify localized PCa without extending through the prostatic capsule or seminal vesicle invasion. Men were scored as 0 according to the International Continence Inquiring Committee's Questionnaire (ICI-Q-SF). The exclusion criteria were patients who were lost to follow-ups. Using the inclusion and exclusion criteria, a total of 263 cases were finally recruited in this study. The study was approved by the Ethics Committee of Shanghai Ruijin Hospital, and written informed consent was obtained from all patients.

Preoperative baseline data of the patients, operation time, estimated blood loss (EBL), postoperative complications, and 1-, 3-, 6- and 12-month postoperative UC were collected retrospectively. Pad-free UC at catheter removal, 1- and 3-, 6and 12-month was followed up by telephone interviews that included questions about pad usage and duration of incontinence. UC recovery was defined as no pad. Histopathological analysis was processed according to the recommendations of the American Society of Clinical Pathologists (ASCP) (9).

\section{Surgical Technique}

Patients underwent either RALRP or LRP. Pelvic lymph node dissection (PLND) was performed in patients with risk of LN involvement $>5 \%$ in the Briganti nomogram (10).

\section{Key Steps of the Combined Technique Are Reported Below}

Patients underwent a transperitoneal six-port robotic surgery or traditional LRP. Robotic/laparoscopy set-up and port placement 
followed the techniques described by previous studies $(11,12)$. Some steps and anatomical landmarks of our modified technique are illustrated in Figure 1.

\section{Bladder Neck Transection}

The assistant pulled the Foley catheter back and forth; this simple maneuver aided in the identification of the bladder neck. We approach the bladder neck directly without incising the endopelvic fascia or ligating the dorsal venous complex (DVC), (Figure 1A).

\section{Preservation of the DVC}

We transected the anterior bladder neck without ligating the DVC, which was followed by posterior bladder neck dissection and posterior plane dissection between the prostate and the rectum. This plane was incised precisely; after seminal vesicle release and vas deferens transection, both the vasa and seminal vesicle were then grasped, the posterior prostate was lifted, and the anterior layer of Denonvilliers fascia was well protected. An incision was made between the posterior capsule of prostate and the anterior layer of Denonvilliers fascia, (Figure 1B).

\section{Intrafascial Prostate Dissection}

We avoided incising the prostatic fascia anteriorly, where the fascia was fused with the puboprostatic ligament and covered the dorsal venous plexus. The nerve-sparing technique was performed by starting at the 5- and 7-o'clock position of the posterolateral region; developing an avascular plane between the prostatic pseudocapsule and the prostate fascia; continuing with blunt and cold dissection toward the anterior and distal surface of the prostate, following the intrafascial plane by using the retrograde method; maximizing the preservation of the neurovascular bundle (NVB) complex until reaching the prostatic anterior fibromuscular stroma, (Figure 1C). When performed properly, curtains of periprostatic tissue hang from the pubourethral ligament.
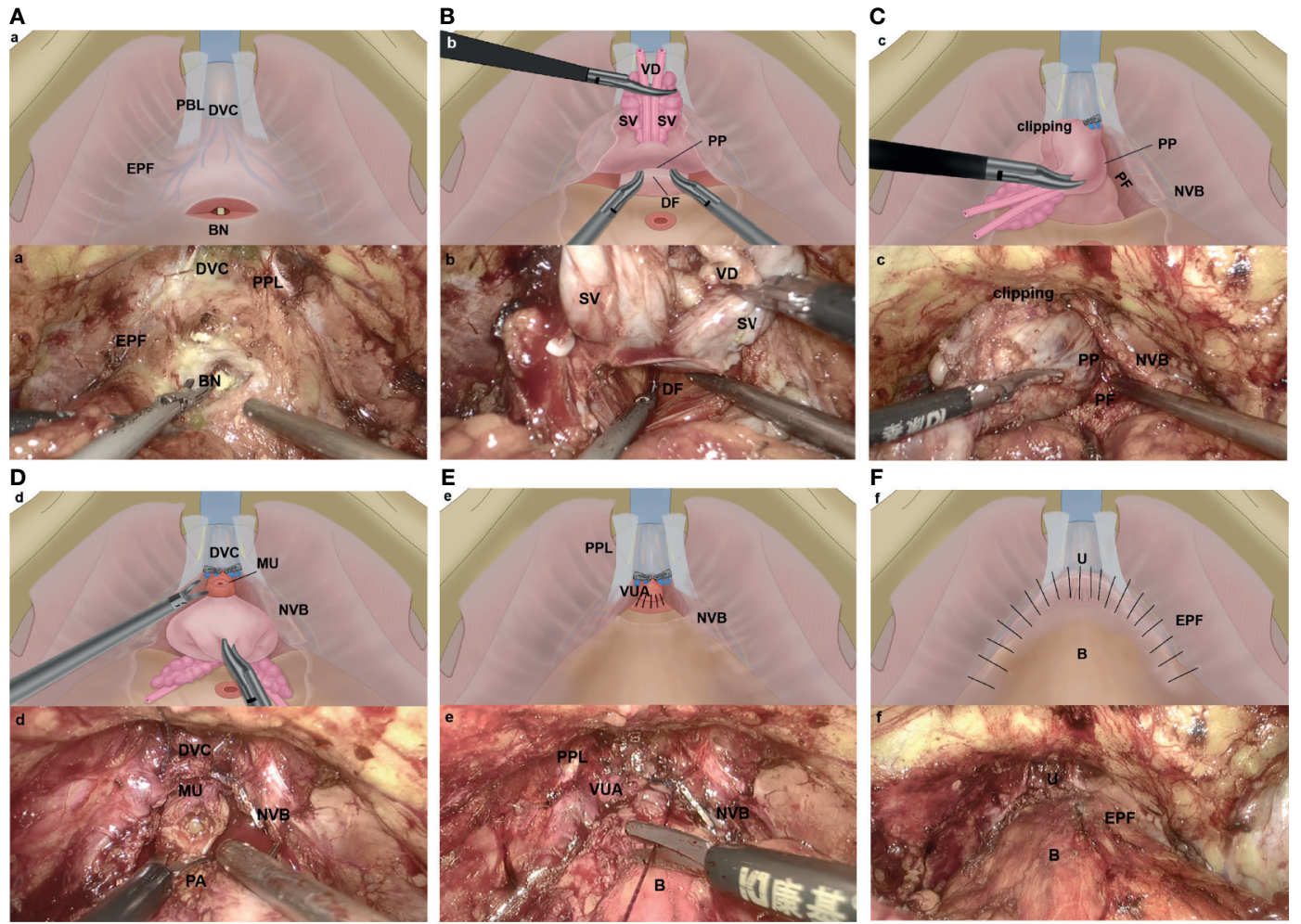

FIGURE 1 | Key steps of the Com-miRP technique (A-F). (A) The anterior bladder neck was incised without incising the endopelvic fascia or ligating the dorsal venous complex. EPF, endopelvic fascia; BN, bladder neck; DVC, dorsal vascular complex; PPL, puboprostatic ligaments. (B) The seminal vesicle release and vas deferens are transected precisely, and the anterior layer of Denonvilliers fascia is well protected. An incision is made between the posterior capsule of prostate and the anterior layer of Denonvilliers fascia. DF, Denonvillier's fascia; SV, seminal vesicle; VD, vas deferens; PP, prostatic pseudocapsule. (C) By starting at the 5- and 7o'clock position, we develop an avascular plane between prostatic pseudocapsule and prostate fascia by using the retrograde method. The preservation of the NVB complex is maximized. The DVC is controlled by clipping temporarily in case of bleeding. PP, prostatic pseudocapsule; NVB, neurovascular bundle; PF, prostatic fascial. (D) Apical dissection is performed underneath the DVC. The apical dissection is minimized, and the distal urethral sphincter is preserved by retracting the prostate firmly to the head of the patient. The urethra is then transected sharply $5 \mathrm{~mm}$ distal to the apical prostate. PA, prostatic apex; NVB, neurovascular bundle; DVC, dorsal vascular complex; MU, membranous urethral. (E) Vesicourethral anastomosis is performed with the technology described by Mani Menon et al. (9) PPL, puboprostatic ligaments; VUA, vesicourethral anastomosis; NVB, neurovascular bundle; B, bladder. (F) The puboprostatic ligaments, parietal endopelvic fascia, and anterior detrusor apron are reconstructed on both sides to support the anastomosis. U, urethra; B, bladder; EPF, endopelvic fascia. 
Apical Dissection Technique and Control Of DVC Bleeding Apical dissection was performed underneath the DVC, avoiding injury to the anterior vascular structures. After the prostatic fascia was dissected off the prostatic apex, we minimized the apical dissection and preserved the distal urethral sphincter by retracting the prostate firmly to the head of the patient. The urethra was then transected sharply $5 \mathrm{~mm}$ distal to the apical prostate, (Figure 1D). The DVC was controlled by temporary titanium clipping or selective stitch in case of bleeding, avoiding a deep stitch of the DVC and circumjacent musculofascial tissue. We also tried to protect the cavernosal nerves, which were close to the urethra and were vulnerable to thermal or traction trauma.

\section{Urethrovesical Anastomosis and Anterior Reconstruction}

A running 2-0 suture (Prolene; Ethicon) was used for the urethrovesical anastomosis, (Figure 1E). We used the technique described by Mani Menon (9). Once the integrity of the anastomosis was identified, the puboprostatic ligaments, parietal endopelvic fascia, and anterior detrusor apron were reconstructed on both sides to support the anastomosis. After the anterior reconstruction was completed, the former clippings which were used to control DVC were removed simultaneously, (Figure 1F).

Comparatively speaking, Conv-RP was performed in accordance with the NHS routine practice. The endopelvic fascia was incised. The DVC was processed with distal bunching and cut off with an electrosurgical scalpel. As a result, bilateral puboprostatic ligaments were not preserved in Conv-RP group.

Both in the Conv-RP group and Comb-RP group, the drain was removed on days $2-3$ postoperatively, and patients were discharged with an indwelling Foley catheter. The catheter was removed on day 14 after surgery.

\section{Statistical Analysis}

SPSS version 22.0 (IBM Corp, Armonk, NY, USA) was used for statistical analysis. Normally distributed continuous variables were reported as means with standard deviations (SDs) and tested by Student's t-test. Non-normally distributed continuous variables were reported as medians with interquartile range (IQR) and tested by Mann-Whitney $\mathrm{U}$ test. For categorical variables, counts and percentages were calculated. Categorical variables in two surgical groups were tested by Chi-square test and Fisher's exact tests, as appropriate. Kaplan-Meier analyses were used to compare the times to pad-free continence between the Comb-RP and Conv-RP groups. Scatter-graphs of perioperative, functional, and oncological outcomes were drawn using locally weighted scatterplot smoothing (LOWESS), and a plateau phase was estimated. A two-tailed test with $\mathrm{p}<0.05$ was considered statistically significant.

\section{RESULTS}

A total of 263 RPs were performed, including 173 Conv-RPs and 90 Comb-RPs. The median follow-up time was 21 months. There were fewer patients who underwent robotic surgery in Comb-RP than in Conv-RP. There were no significant differences in age, BMI, American Society of Anesthesiologists (ASA) classification, PSA level, lower urinary tract symptom (LUTS), and prostate volume between the two groups (Table 1).

\section{Intra-Operative and Oncological Outcomes}

As shown in Table 2, the length of operation time in Conv-RP group was longer than that in Comb-RP group (125 vs. $110 \mathrm{~min}, \mathrm{P}=0.011$ ). The distribution of pathological T (pT) stage in Conv-RP and Comb$\mathrm{RP}$ was slightly different, and patients in Conv-RP group tended to have early $\mathrm{pT}$ stage. There were no significant differences in estimated blood loss (EBL), International Society of Urological Pathology (ISUP) group, positive surgical margin (PSM), and postoperative complication between the two groups. Prostate margin status was divided into four groups: negative margin, apical PSM, non-apical PSM, and multiple PSM (Table 3): 8 (4.6\%) patients in Conv-RP group vs. 2 (2.2\%) in Comb-RP group had positive apical margin, 33

TABLE 1 | Baseline parameters of patients undergoing Conv-RP or Comb-RP.

\begin{tabular}{|c|c|c|c|}
\hline Characteristics & Conv-RP(n = 173) & Comb-RP(n = 90) & $P$ \\
\hline $\mathrm{BMI}, \mathrm{kg} / \mathrm{m}^{2}$ (mean $\left.\pm \mathrm{SD}\right)$ & $24.6 \pm 2.9$ & $24.2 \pm 2.8$ & $0.370^{\mathrm{a}}$ \\
\hline 1 & $3(1.7)$ & 3 (3.3) & \\
\hline 2 & $127(73.4)$ & 75 (83.3) & \\
\hline 3 & 43 (24.9) & 12 (13.3) & \\
\hline Median PSA ng/ml (IQR) & $10.8(7.5-16.1)$ & $10.8(7.5-15.4)$ & $0.678^{b}$ \\
\hline Robotic surgery, n (\%) & $35(20.2)$ & $9(10.0)$ & $0.035^{c}$ \\
\hline PLND, (\%) & 89 (51.4) & $38(42.2)$ & 0.156 \\
\hline
\end{tabular}

astudent's t-test.

${ }^{b}$ Mann-Whitney $\cup$ test.

${ }^{c}$ Chi-square test.

${ }^{d}$ Fisher's exact test.

SD, standard deviations; IQR, interquartile range; LUTS, lower urinary tract symptom; RP, radical prostatectomy; Conv-RP, conventional radical prostatectomy; Comb-RP, combined radical prostatectomy; PSA, prostate specific antigen; ASA, American Society of Anesthesiologists; BMI, body mass index; PLND, pelvic lymph node dissection.

Bold values indicate significant $p$-values. 
TABLE 2 | Perioperative and pathological parameters of patients undergoing Conv-RP or Comb-RP.

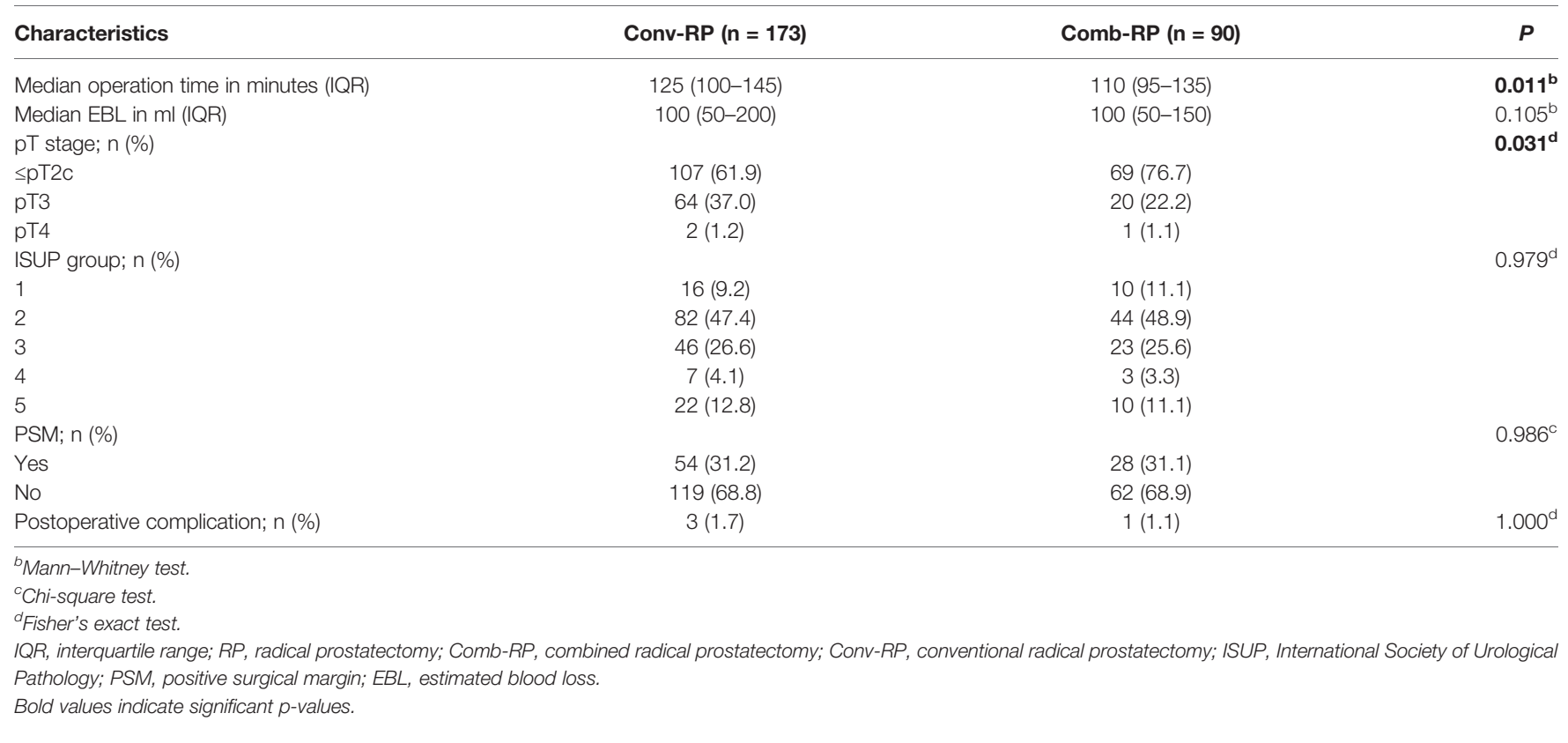

TABLE 3 | Margin details of patients undergoing Conv-RP or Comb-RP.

\begin{tabular}{|c|c|c|c|}
\hline Margin details & Conv-RP $(n=173)$ & Comb-RP $(n=90)$ & $P$ \\
\hline Negative margin; n (\%) & $119(68.8)$ & $62(68.9)$ & $0.986^{\circ}$ \\
\hline Apical PSM; n (\%) & $8(4.6)$ & $2(2.2)$ & $0.984^{\circ}$ \\
\hline Non-apical PSM; n (\%) & $33(19.1)$ & $17(18.9)$ & $0.971^{\mathrm{C}}$ \\
\hline Multiple PSM; n (\%) & $13(7.5)$ & $9(10.0)$ & $0.490^{\circ}$ \\
\hline
\end{tabular}

${ }^{c}$ Chi-square test.

Comb-RP, combined radical prostatectomy; Conv-RP, conventional radical prostatectomy; PSM, positive surgical margin.

(19.1\%) vs. 17 (18.9) patients had a positive margin in non-apical site, $13(7.5 \%)$ vs. $9(10.0 \%)$ patients had positive margins in multiple site, and $119(68.8 \%)$ vs. $62(68.9 \%)$ patients had negative surgical margin. Chi-square test showed no statistical significance between the two groups in all margin status groups.

\section{Functional Outcomes}

The results of comparison of postoperative UC between Conv-RP and Comb-RP were shown in Table 4: instant UC: 17.3 vs. $27.8 \%$ $(P=0.048)$; 1 -month pad-free UC: 35.8 vs. $50.0 \%(P=0.027)$; 3 month pad-free UC: 60.5 vs. $76.1 \%(P=0.012)$; 6-month pad-free UC: 87.7 vs. $96.5 \%(P=0.022)$; 12-month pad-free UC: $94.7 v s$.
97.7\% ( $P=0.343)$. Kaplan-Meier analysis showed significantly higher and faster continence recovery in the Comb-RP group (Log Rank $P=0.001$; Figure 2). The mean time to achieve padfree continence was 4.9 months in the conventional group and 2.6 months in the combined RP group.

\section{Learning Curve Analysis}

The learning curve for operation time decreased as the cases increased, reaching the lowest point at the 50th case (Supplement Figure S1). The learning cure for EBL after surgery showed a pattern similar to operation time. The lowest point of both was attained at the 50th case (Supplement Figure

TABLE 4 | Continence outcomes of patients undergoing Conv-RP or Comb-RP.

\begin{tabular}{|c|c|c|c|}
\hline Continence outcomes & Conv-RP $(n=173)$ & Comb-RP $(n=90)$ & $P$ \\
\hline Instant UC; n (\%) & $30(17.3)$ & $25(27.8)$ & $0.048^{b}$ \\
\hline UC at 1 month; $n(\%)$ & $62(35.8)$ & $45(50.0)$ & $0.027^{b}$ \\
\hline UC at 3 months; n (\%) & $104(60.5)$ & $67(76.1)$ & $0.012^{b}$ \\
\hline UC at 6 months; n (\%) & $150(87.7)$ & $83(96.5)$ & $0.022^{b}$ \\
\hline UC at 12 months; n (\%) & $160(94.7)$ & $84(97.7)$ & $0.343^{c}$ \\
\hline
\end{tabular}

${ }^{b}$ Chi-square test.

${ }^{c}$ Fisher's exact test.

$R P$, radical prostatectomy; Comb-RP, combined radical prostatectomy; Conv- $R P$, conventional radical prostatectomy; UC, urinary continence.

Bold values indicate significant $p$-values. 


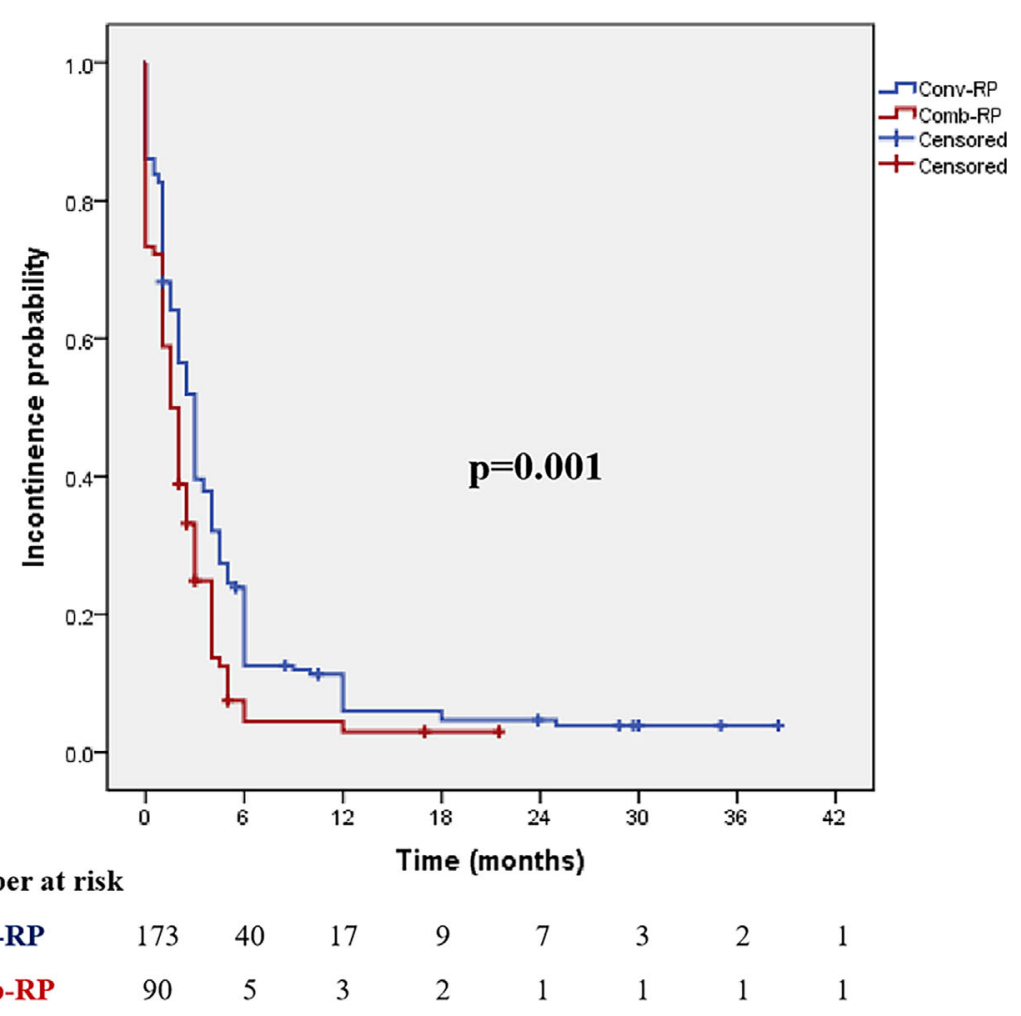

FIGURE 2 | Cumulative probability of incontinence after radical prostatectomy by study groups (Comb-RP and conv-RP). Conv-RP, conventional radical prostatectomy; Comb-RP, combined radical prostatectomy. Log Rank $p=0.001$.

S2). Oncological outcome was reported as PSM rate, which reached a plateau at the 60th case (Supplement Figure S3). In terms of the functional outcomes, the learning curve for instant, 1-, 3-, and 12-month UC reached a plateau at the 30th, 50th, 40th, 20th cases, respectively (Supplement Figure 4).

\section{DISCUSSION}

Although RALRP has improved postoperative UC remarkably as compared with conventional LRP $(13,14)$, urinary incontinence remains the main postoperative complication affecting the quality of life of such patients (15). In the present study, we introduced a new combined technique which helped early UC recovery but showed no significant difference in long-term UC as compared with conventional RP. Our result demonstrated that the Comb-RP technique was safe and feasible as there were no significant differences in the operation time, EBL, PSM rate, and postoperative complications between the Comb-RP group and Conv-RP group. The learning curve also indicated all perioperative, functional, and oncological outcomes reached the lowest point or plateau in an early period.

Using the combined technique, the instant, 1-, 3-, and 6month UC recovery rates improved significantly as compared with Conv-RP. The possible reasons for such improvement can be summarized in the following points.
1. Preservation of the neurovascular structure by using the DVC no-ligation technique, the NVB dissociation and preservation technique without thermal and mechanical damage (8), and the technique to minimize apical dissection (16) so that the vessel and nerve in the apex of prostate can be maximally protected.

2. Preservation of the muscle, fascia, and ligament supporting structures by using the bladder neck-sparing technique (17), membranous urethral length preservation technique (18), and the minimal apical dissection technique (16) to maximally preserve the muscular UC tissue in the bladder neck, the urethra rhabdosphincter in the apex, and the levator ani muscle around the apex, which is considered as important support structure.

3. Anterior reconstruction is important. We sutured the muscular fibers of the bladder neck to the periurethral tissue between the DVC and the anastomosed urethra and realigned the bladder to the pelvic sidewall with the aim to restore the periurethral support and vesicourethral angle.

PSM is recognized as an important factor in biochemical recurrence and disease progression; the PSM rate reported in previous studies is $8.6-33.2 \%(12,19-21)$. The PSM rate in our study was similar with that in previous studies. On the one hand, PSM may be due to the intrafascial resection technique itself (22). On the other hand, PSM may be explained by the heterogenicity of the included patients as the high proportions 
of PCa patients with a pT3a or higher stage. Previous studies suggested that the location, length, and number of PSM are also important (20). The apical PSM represents the most common site after RP, especially miRP (21). However, positive margin at the apex showed less influence than other peri-operative variables, like maximum positive biopsy cores on biochemical recurrence (19). Finding from our analysis confirmed that the non-apical site is the most frequent location of PSM; differences of PSM location likely reflect variations in surgical technique. However, we did not find any significant difference in terms of PSM between patients in Comb-RP group and those in Conv-RP group.

Understanding the learning curve of a new surgical technique is very important for surgical education. To date, some studies have reported the learning curves of LRP and RALRP. An early study reported the safety and efficacy of Retzius-sparing RALRP, and there was a linear relationship between surgery experience and imitated UC in the first 50 cases, without reaching a plateau (23). Some authors asserted that with $>100$ cases, the plateau on early continence is achievable (24). Regarding PSMs, the evidence available is controversial: some researchers did not find improvement of the PSM rate during the learning curve $(23,25)$, while others, in a single surgeon series, found an overall reduction of positive margins with the amount of experience (26). In an early series about traditional RARP, Patel and colleagues (27) showed that operative times and EBL are reduced with the experience of the surgeon. It has been demonstrated that longer surgical experience also decreases complications. In this study, while PSM rate was stable as the cases increased, operation time, EBL and LOS after surgery gradually increased after the lowest point. Possible explanations are as follows: first, there was a tendency to perform more difficult cases while the experience increased. Second, we tried new technique in patients with intermediate to high-risk PCa. Finally, more robotic surgeries were performed in recent years, and the surgeon needed more time to transfer from a laparoscopic environment to using a robotic interface.

Porpiglia et al. (28) reported 5-year functional and oncological outcomes of their previously published prospective randomized study comparing robot-assisted radical prostatectomy (RARP) and laparoscopic radical prostatectomy (LRP). Their results suggested that the probability of achieving continence [odds ratio (OR) 2.47, $\mathrm{p}<0.021$ ] and potency (OR $2.35, \mathrm{p}<0.028$ ) over time was more than doubled for the RARP compared to the LRP group. In our study, $20.2 \%$ of patients in Conv-RP group underwent RARP, while only $10.0 \%$ in CombRP group selected robotic surgery. However, the Comb-RP group finally achieved better functional outcomes with less proportion of patients who underwent robotic surgery, which fully suggested our Com-RP technology has a significant positive influence on UC.

The main limitation of our data arises from its retrospective design. The study also has some other limitations. First, it is necessary to conduct follow-up study to trace the long-term oncologic outcome to ascertain the impact of this technique on tumor control. Second, it is still necessary to obtain data from randomized prospective studies to support our conclusions. Third, this technique should be used with caution in high-risk PCa patients according to the concrete situation. It is relatively safe in tumor control of low- and intermediate-risk PCa patients in the context of high-quality research data currently available $(29,30)$.

\section{CONCLUSION}

This study was based on the DUSC anatomic theory, and the results have demonstrated that the new technique is a relatively safe, effective, and reliable technique for tumor control and early UC in RP. Additionally, learning curves of peri-operative outcomes including operation time and $\mathrm{EBL}$ achieved the lowest point during a short learning process. UC and PSM rates also attained a plateau at a similar period of time.

\section{DATA AVAILABILITY STATEMENT}

The raw data supporting the conclusions of this article will be made available by the authors, without undue reservation.

\section{ETHICS STATEMENT}

The studies involving human participants were reviewed and approved by the Ethics Review Board of Ruijin Hospital. The patients/participants provided their written informed consent to participate in this study.

\section{AUTHOR CONTRIBUTIONS}

All authors contributed to the article and approved the submitted version. DX, LC, and AL designed the research study. XY, YG, and $\mathrm{HH}$ contributed essential reagents or tools. AL and $\mathrm{WL}$ analyzed the data. AL and LC wrote the paper.

\section{FUNDING}

This study was funded by National Natural Science Foundation of China (No. 81972405); Shanghai Committee of Science and Technology, China (No. 18411960100; No.20Y11904700).

\section{SUPPLEMENTARY MATERIAL}

The Supplementary Material for this article can be found online at: https://www.frontiersin.org/articles/10.3389/fonc.2021.711093/ full\#supplementary-material 


\section{REFERENCES}

1. Egan J, Marhamati S, Carvalho FLF, Davis M, O’Neill J, Lee H, et al. RetziusSparing Robot-Assisted Radical Prostatectomy Leads to Durable Improvement in Urinary Function and Quality of Life Versus Standard Robot-Assisted Radical Prostatectomy Without Compromise on Oncologic Efficacy: Single-Surgeon Series and Step-By-Step Guide. Eur Urol (2021) 79 (6):839-57. doi: 10.1016/j.eururo.2020.05.010

2. Porpiglia F, Bertolo R, Manfredi M, De Luca S, Checcucci E, Morra I, et al. Total Anatomical Reconstruction During Robot-Assisted Radical Prostatectomy: Implications on Early Recovery of Urinary Continence. Eur Urol (2016) 69(3):485-95. doi: 10.1016/j.eururo.2015.08.005

3. Nyarangi-Dix JN, Radtke JP, Hadaschik B, Pahernik S, Hohenfellner M. Impact of Complete Bladder Neck Preservation on Urinary Continence, Quality of Life and Surgical Margins After Radical Prostatectomy: A Randomized, Controlled, Single Blind Trial. J Urol (2013) 189(3):891-8. doi: 10.1016/j.juro.2012.09.082

4. Schlomm T, Heinzer H, Steuber T, Salomon G, Engel O, Michl U, et al. Full Functional-Length Urethral Sphincter Preservation During Radical Prostatectomy. Eur Urol (2011) 60(2):320-9. doi: 10.1016/j.eururo.2011.02.040

5. Patel VR, Coelho RF, Palmer KJ, Rocco B. Periurethral Suspension Stitch During Robot-Assisted Laparoscopic Radical Prostatectomy: Description of the Technique and Continence Outcomes. Eur Urol (2009) 56(3):472-8. doi: 10.1016/j.eururo.2009.06.007

6. Joshi N, de Blok W, van Muilekom E, van der Poel H. Impact of Posterior Musculofascial Reconstruction on Early Continence After Robot-Assisted Laparoscopic Radical Prostatectomy: Results of a Prospective Parallel Group Trial. Eur Urol (2010) 58(1):84-9. doi: 10.1016/j.eururo.2010.03.028

7. Cochetti G, Boni A, Barillaro F, Pohja S, Cirocchi R, Mearini E. Full Neurovascular Sparing Extraperitoneal Robotic Radical Prostatectomy: Our Experience With PERUSIA Technique. J Endourol (2017) 31(1):32-7. doi: 10.1089/end.2016.0477

8. Covas Moschovas M, Bhat S, Onol FF, Rogers T, Roof S, Mazzone E, et al. Modified Apical Dissection and Lateral Prostatic Fascia Preservation Improves Early Postoperative Functional Recovery in Robotic-Assisted Laparoscopic Radical Prostatectomy: Results From a Propensity Score-Matched Analysis. Eur Urol (2020) 78(6):875-84. doi: 10.1016/j.eururo.2020.05.041

9. True LD. Surgical Pathology Examination of the Prostate Gland. Practice survey by American society of clinical pathologists. Am J Clin Pathol (1994) 102(5):572-9. doi: 10.1093/ajcp/102.5.572

10. Briganti A, Larcher A, Abdollah F, Capitanio U, Gallina A, Suardi N, et al. Updated Nomogram Predicting Lymph Node Invasion in Patients With Prostate Cancer Undergoing Extended Pelvic Lymph Node Dissection: The Essential Importance of Percentage of Positive Cores. Eur Urol (2012) 61 (3):480-7. doi: 10.1016/j.eururo.2011.10.044

11. Türk I, Deger S, Winkelmann B, Schönberger B, Loening SA. Laparoscopic Radical Prostatectomy. Technical Aspects and Experience With 125 Cases. Eur Urol (2001) 40(1):46-52; discussion 53. doi: 10.1159/000049748

12. de Carvalho PA, Barbosa J, Guglielmetti GB, Cordeiro MD, Rocco B, Nahas WC, et al. Retrograde Release of the Neurovascular Bundle With Preservation of Dorsal Venous Complex During Robot-Assisted Radical Prostatectomy: Optimizing Functional Outcomes. Eur Urol (2020) 77(5):628-35. doi: 10.1016/j.eururo.2018.07.003

13. Cao L, Yang Z, Qi L, Chen M. Robot-Assisted and Laparoscopic vs Open Radical Prostatectomy in Clinically Localized Prostate Cancer: Perioperative, Functional, and Oncological Outcomes: A Systematic Review and Meta-Analysis. Med (Baltimore) (2019) 98(22):e15770. doi: 10.1097/md.0000000000015770

14. Basiri A, de la Rosette JJ, Tabatabaei S, Woo HH, Laguna MP, Shemshaki H. Comparison of Retropubic, Laparoscopic and Robotic Radical Prostatectomy: Who is the Winner? World J Urol (2018) 36(4):609-21. doi: 10.1007/s00345-018-2174-1

15. Hoffman KE, Penson DF, Zhao Z, Huang LC, Conwill R, Laviana AA, et al. Patient-Reported Outcomes Through 5 Years for Active Surveillance, Surgery, Brachytherapy, or External Beam Radiation With or Without Androgen Deprivation Therapy for Localized Prostate Cancer. JAMA (2020) 323 (2):149-63. doi: 10.1001/jama.2019.20675

16. Bianchi L, Turri FM, Larcher A, De Groote R, De Bruyne P, De Coninck V, et al. A Novel Approach for Apical Dissection During Robot-Assisted Radical Prostatectomy: The "Collar" Technique. Eur Urol Focus (2018) 4(5):677-85. doi: 10.1016/j.euf.2018.01.004
17. Nyarangi-Dix JN, Tichy D, Hatiboglu G, Pahernik S, Tosev G, Hohenfellner M. Complete Bladder Neck Preservation Promotes Long-Term PostProstatectomy Continence Without Compromising Midterm Oncological Outcome: Analysis of a Randomised Controlled Cohort. World J Urol (2018) 36(3):349-55. doi: 10.1007/s00345-017-2134-1

18. Jo JK, Hong SK, Byun SS, Zargar H, Autorino R, Lee SE. Positive Surgical Margin in Robot-Assisted Radical Prostatectomy: Correlation With Pathology Findings and Risk of Biochemical Recurrence. Minerva Urol Nefrol (2017) 69 (5):493-500. doi: 10.23736/s0393-2249.17.02707-2

19. Kohjimoto Y, Yamashita S, Kikkawa K, Iba A, Matsumura N, Hara I. The Association of Length of the Resected Membranous Urethra With Urinary Incontinence After Radical Prostatectomy. Urol J (2020) 17(2):146-51. doi: 10.22037/uj.v0i0.4753

20. Phukan C, McLean A, Nambiar A, Mukherjee A, Somani B, Krishnamoorthy R, et al. Retzius Sparing Robotic Assisted Radical Prostatectomy vs. Conventional Robotic Assisted Radical Prostatectomy: A Systematic Review and Meta-Analysis. World J Urol (2020) 38(5):1123-34. doi: 10.1007/s00345-019-02798-4

21. Falzarano SM, Nyame YA. Clinicopathologic Features and Outcomes of AnteriorDominant Prostate Cancer: Implications for Diagnosis and Treatment. Prostate Cancer Prostatic Dis (2020) 23(3):435-40. doi: 10.1038/s41391-019-0199-1

22. Preston MA, Breau RH, Lantz AG, Morash C, Gerridzen RG, Doucette S, et al. The Association Between Nerve Sparing and a Positive Surgical Margin During Radica Prostatectomy. Urol Oncol (2015) 33(1):18.e1-6. doi: 10.1016/j.urolonc.2014.09.006

23. Galfano A, Secco S. Retzius-Sparing Robot-Assisted Radical Prostatectomy: Early Learning Curve Experience in Three Continents. BJU Int (2021) 127 (4):412-7. doi: 10.1111/bju.15196

24. Good DW, Stewart GD, Laird A, Stolzenburg JU, Cahill D, McNeill SA. A Critical Analysis of the Learning Curve and Postlearning Curve Outcomes of Two Experience- and Volume-Matched Surgeons for Laparoscopic and Robot-Assisted Radical Prostatectomy. J Endourol (2015) 29(8):939-47. doi: 10.1089/end.2014.0810

25. Jaulim A, Srinivasan A, Hori S, Kumar N, Warren AY, Shah NC, et al. A Comparison of Operative and Margin Outcomes From Surgeon Learning Curves in Robot Assisted Radical Prostatectomy in a Changing Referral Practice. Ann R Coll Surg Engl (2018) 100(3):226-9. doi: 10.1308/rcsann.2018.0001

26. Islamoglu E, Karamik K, Ozsoy C, Tokgoz H, Ates M, Savas M. The Learning Curve Does Not Affect Positive Surgical Margin Status in Robot-Assisted Laparoscopic Prostatectomy. Urol J (2018) 15(6):333-8. doi: 10.22037/uj.v0i0.4426

27. Patel VR, Tully AS, Holmes R, Lindsay J. Robotic Radical Prostatectomy in the Community Setting-the Learning Curve and Beyond: Initial 200 Cases. J Urol (2005) 174(1):269-72. doi: 10.1097/01.ju.0000162082.12962.40

28. Porpiglia F, Fiori C, Bertolo R, Manfredi M, Mele F, Checcucci E, et al. FiveYear Outcomes for a Prospective Randomised Controlled Trial Comparing Laparoscopic and Robot-Assisted Radical Prostatectomy. Eur Urol Focu (2018) 4(1):80-6. doi: 10.1016/j.euf.2016.11.007

29. Walz J, Epstein JI, Ganzer R, Graefen M, Guazzoni G, Kaouk J, et al. A Critical Analysis of the Current Knowledge of Surgical Anatomy of the Prostate Related to Optimisation of Cancer Control and Preservation of Continence and Erection in Candidates for Radical Prostatectomy: An Update. Eur Urol (2016) 70(2):301-11. doi: 10.1016/j.eururo.2016.01.026

30. Martini A, Falagario UG, Villers A, Dell'Oglio P, Mazzone E, Autorino R, et al. Contemporary Techniques of Prostate Dissection for Robot-Assisted Prostatectomy. Eur Urol (2020) 78(4):583-91. doi: 10.1016/j.eururo.2020.07.017

Conflict of Interest: The authors declare that the research was conducted in the absence of any commercial or financial relationships that could be construed as a potential conflict of interest.

Publisher's Note: All claims expressed in this article are solely those of the authors and do not necessarily represent those of their affiliated organizations, or those of the publisher, the editors and the reviewers. Any product that may be evaluated in this article, or claim that may be made by its manufacturer, is not guaranteed or endorsed by the publisher.

Copyright (C) 2021 Liu, Gao, Huang, Yang, Lin, Chen and Xu. This is an open-access article distributed under the terms of the Creative Commons Attribution License (CC BY). The use, distribution or reproduction in other forums is permitted, provided the original author(s) and the copyright owner(s) are credited and that the original publication in this journal is cited, in accordance with accepted academic practice. No use, distribution or reproduction is permitted which does not comply with these terms. 